\title{
Intermittent, subtle change of ST segment in narrow QRS regular tachycardia
}

Hyeong Won Seo, Hyung Wook Park, Ki Hong Lee, Joon-Ho Ahn, Ji Eun Yu, Nam Sik Yoon, and Jeong Gwan Cho

Department of Cardiovascular Medicine, Chonnam National University Hospital, Gwangju, Korea

Received: April 16, 2015

Revised : June 16, 2015

Accepted: June 24, 2015

\section{Correspondence to}

Hyung Wook Park, M.D.

Department of Cardiovascular Medicine, Chonnam National

University Hospital, 42 Jebong-ro,

Dong-gu, Gwangju 61469, Korea

Tel: $+82-62-220-6572$

Fax: +82-62-223-3105

E-mail:mdhwp@naver.com
To the Editor,

A 54-year-old man presented with frequent episodes of sudden palpitation. Twelve-lead electrocardiogram demonstrated narrow QRS complex tachycardia at a rate of $150 / \mathrm{min}$. The initial part of the ST segment occasionally became deeper and sharper during the tachycardia (arrow, Fig. 1). A cardiac electrophysiological study was performed and the narrow QRS complex tachycardia was reproducibly induced by a single atrial extra-stimulus. The tachycardia intermittently changed at the initial part of the ST segment (arrow, Fig. 2A). Atrial activity at the high right atrium (HRA) always preceded those of the His bundle and coronary sinus (CS) during the first induced supraventricular tachycardia (SVT) with an atrial cycle length of $344 \mathrm{~ms}$, but the retrograde atrial activation sequence in the CS electrogram changed occasionally. Simultaneous activation of the right anterolateral and left lateral accessory pathways (APs) deepened and sharpened the initial point of the ST segment compared to right anterolateral AP activation alone. Radiofrequency catheter ablation abolished conduction over the right side AP. A single ventricular extra-stimulus induced a second tachycardia (388 ms) with the earliest, consistent retrograde atrial activity at the distal CS (Fig. 2B). The earlier retrograde atrial activation at HRA and ST segment change were not present after radiofrequency catheter ablation of the right anterolateral AP. The exact locations of both APs are described in Fig. $3 \mathrm{~A}$ and $3 \mathrm{~B}$. We ablated the second AP at the left lateral wall using a transaortic approach. Ventriculoatrial conduction occurred through the atrioventricular node after catheter ablation of the two APs.

Electrocardiographic or electrophysiologic findings that demonstrate the presence of multiple AP include (1) changing antegrade delta waves; (2) evidence of multiple retrograde atrial activation routes; and (3) orthodromic SVT with intermittent antegrade fusion complexes [1].

Concealed conduction through another pathway may not be apparent until the dominant pathway is ablated [2]. Detecting multiple APs requires detailed atrial mapping during programmed stimulation, and elimination of an apparent $\mathrm{AP}$ [3]. If the $\mathrm{V}$-A interval or P-wave morphology changes during orthodromic tachycardia, the presence of additional AP should be suspected $[4,5]$. Although a single, fixed retrograde atrial activation pattern is observed in the presence of two or more AP, the presence of different retrograde atrial activation patterns documents the presence of multiple AP [1].

In our case, an additional AP was 

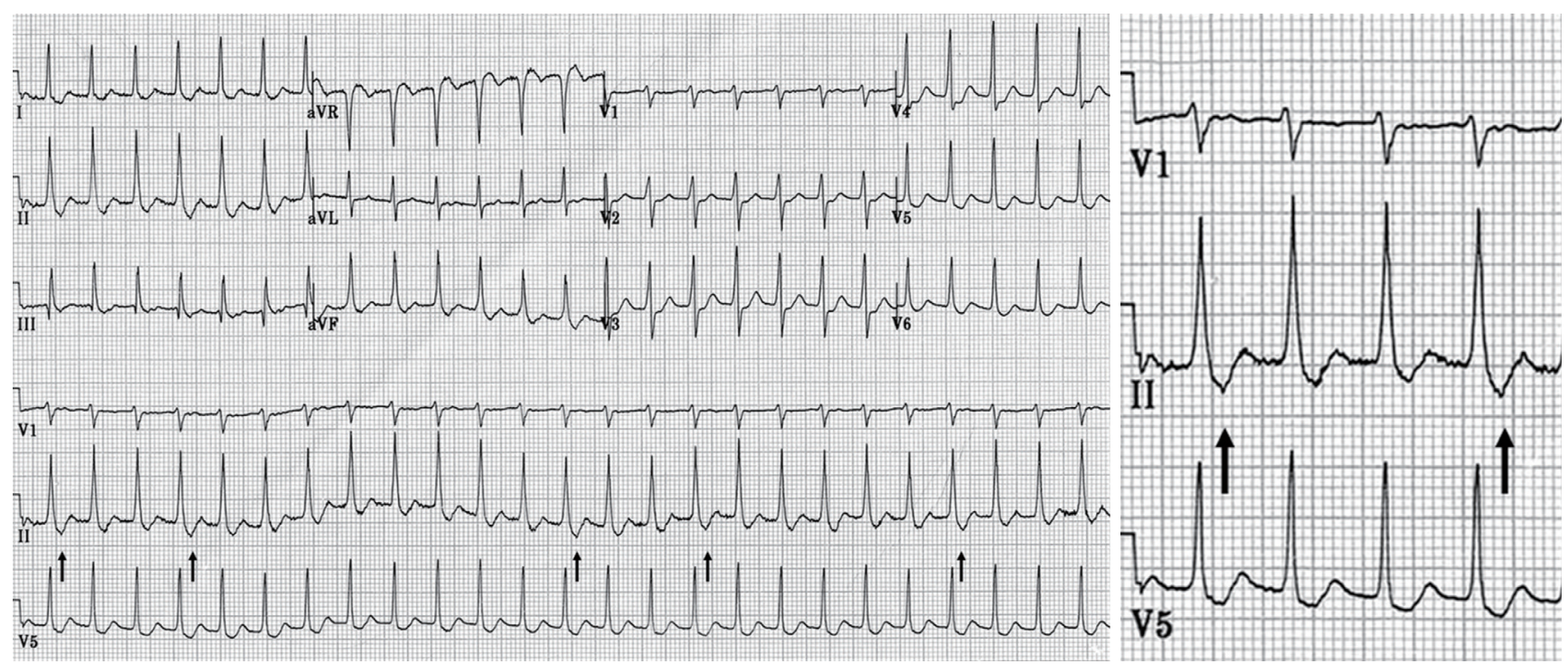

A

B

Figure 1. Twelve-lead surface electrocardiogram $(25 \mathrm{~mm} / \mathrm{sec}, 10 \mathrm{~mm} / \mathrm{mV})$ during a spontaneous episode of paroxysmal tachycardia and four magnified beats of tachycardia (A, B). The initial part of the ST segment of the first and fourth beats (arrows) during tachycardia became sharper and deeper in lead II, and a subtle change was detected in lead V1.

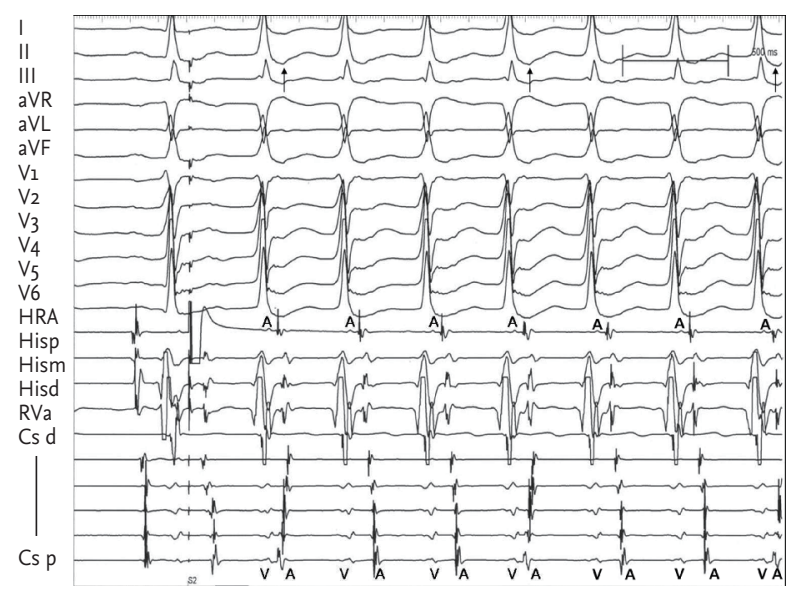

A

Figure 2. (A) Surface electrocardiogram (ECG) and intracardiac electrograms from the high right atrium (HRA), His bundle region (His), right ventricular apex (RVa), and coronary sinus (Cs) during the first induced supraventricular tachycardia (SVT) with a cycle length of $344 \mathrm{~ms}$ showed occasional changes in the retrograde atrial activation sequence in the CS. The initial part of the ST segment on the surface ECG became deeper and sharper when the left-side accessory pathway was activated (arrows). (B) Surface ECG and intracardiac electrograms during the second induced SVT with a cycle length of $383 \mathrm{~ms}$ show a consistent retrograde atrial activation sequence. $\mathrm{V}$, ventricle; $\mathrm{A}$, atrium.

recognized by the appearance of more than one atrial breakthrough point during the first induced tachycardia. Intermittent changes in the retrograde atrial activation sequence occurred in the CS (from distal-to-proximal to proximal-to-distal), whereas retrograde atrial activation in the HRA was the earliest. The consistent earliest activation in the HRA suggests that clinical SVT uses the right anterolateral AP as a re-entry circuit and intermittently the left lateral AP as a bystander. Atrial fusion due to intermittent, simultaneous activation of the two AP changed 

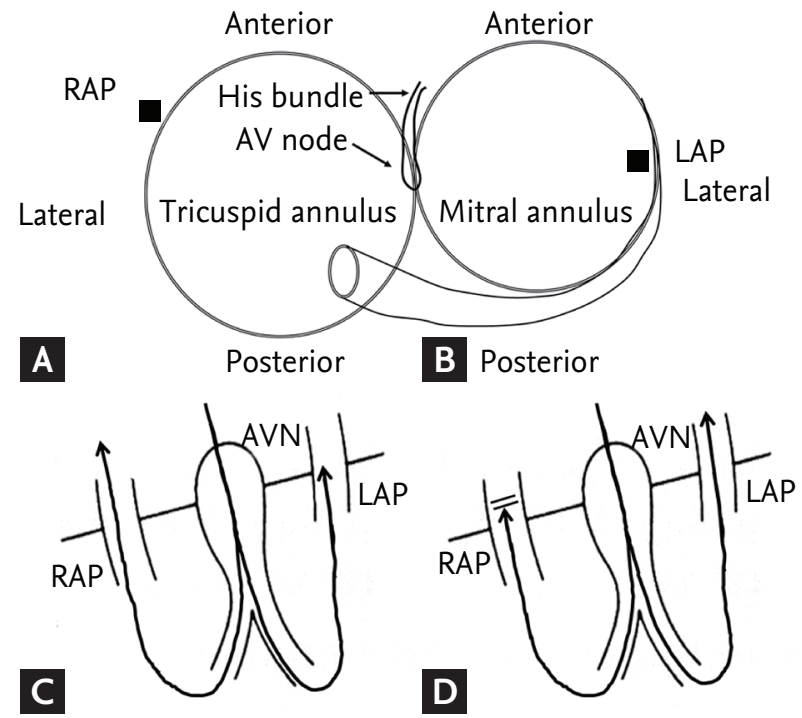

Figure 3. (A, B) Proposed locations of the two accessory pathways (AP) and (C, D) their contribution to tachycardia. (C) The clinical SVT used the right anterolateral AP as a re-entry circuit and intermittently used the left lateral AP as a bystander but conduction through the two pathways created different ST segment morphology. (D) Ablation of the right AP resulted in consistent conduction over the left side AP. RAP, right accessory pathway; LAP, left accessory pathway; AV, atrioventricular; AVN, atrioventricular node.

the initial point of the ST segment on the surface electrocardiogram (Fig. ${ }_{3} \mathrm{C}$ and $3 \mathrm{D}$ ). Careful attention to the ST segment during tachycardia could help identify mul- tiple AP before an electrophysiologic study.

Keywords: ST segment; Electrocardiography; Accessory atrioventricular bundle

\section{Conflict of interest}

No potential conflict of interest relevant to this article was reported.

\section{REFERENCES}

1. Josephson ME. Clinical Cardiac Electrophysiology: Techniques and Interpretations. 3rd ed. Philadelphia: Lippincott Williams and Wilkins, 2002.

2. Chen SA, Hsia CP, Chiang CE, et al. Reappraisal of radiofrequency ablation of multiple accessory pathways. Am Heart J 1993;125:760-771.

3. Heddle WF, Brugada P, Wellens HJ. Multiple circus movement tachycardias with multiple accessory pathways. J Am Coll Cardiol 1984;4:168-175.

4. Obel OA, Camm AJ. Accessory pathway reciprocating tachycardia. Eur Heart J 1998;19 Suppl E:E13-E24, E50-E51.

5. Wellens HJ, Atie J, Smeets JL, Cruz FE, Gorgels AP, Brugada $\mathrm{P}$. The electrocardiogram in patients with multiple accessory atrioventricular pathways. J Am Coll Cardiol 1990;16:745-751. 